\title{
Nanoemulsions of Hydroxysafflor Yellow A for Enhanced Brain Delivery in Cerebral Ischemia Reperfusion-injured mice: Physicochemical and In Vivo Performances
}

\section{Qiong Wang}

Chongqing Medical University

\section{Cailing Zhong}

Chongqing Medical University

Jingqing Zhang

Chongqing Medical University

Hua Zhao

Chongqing Medical University

\section{Yuru Huang}

Chongqing Medical University

Dezhang Zhao

Chongqing Medical University

Junqing Yang ( $\Delta$ cqyangjq@cqmu.edu.cn )

Chongqing Medical University https://orcid.org/0000-0002-7347-6718

\section{Research Article}

Keywords: Hydroxysafflor yellow A, Oral administration, Nanoparticle, Cerebral ischemia-reperfusion injury

Posted Date: October 18th, 2021

DOl: https://doi.org/10.21203/rs.3.rs-968897/v1

License: (c) (i) This work is licensed under a Creative Commons Attribution 4.0 International License. Read Full License 


\section{Abstract}

Stroke has always been a disease threatening human life and health worldwide. Here, we synthesized a new type of hyaluronic acid-modified multi-walled carbon nanotube. Then we prepared hydroxysafflor yellow A-hydroxypropyl- $\beta$-cyclodextrin phospholipid complex water-in-oil nanoemulsion with hyaluronic acid-modified multi-walled carbon nanotubes and chitosan (HC@HMC) for oral treatment of ischemic stroke. First, we measured the intestinal absorption and pharmacokinetics of HC@HMC in rats. We found that the intestinal absorption and the pharmacokinetic behavior of HC@HMC is better than HYA. Then we detected intracerebral concentrations after oral administration of HC@HMC and found that more HYA crossed the blood-brain barrier (BBB). Finally, we evaluated the efficacy of HC@HMC in middle cerebral artery occlusion/reperfusion (MCAO/R)-treated mice. In MCAO/R mice, oral administration of HC@HMC demonstrated a significant protection against cerebral ischemia-reperfusion injury (CIRI). And we also found HC@HMC may exert a protective effect on cerebral ischemia-reperfusion injury through COX2/PGD2/DPs pathway. These results suggest that oral administration of HC@HMC may be a new strategy for the treatment of stroke.

\section{Introduction}

Stroke is a brain disease that generates devastating neurological delcits and is a major public health challenge, given its high mortality and adult disability worldwide ${ }^{[1]}$. Stroke is divided into hemorrhagic stroke(cerebral blood vessels are ruptured) and ischemic stroke (cerebral blood vessels are blocked), of which ischemic stroke accounts for about $87 \%$ of the total incidence of stroke ${ }^{[2]}$. Ischemic stroke is a kind of cerebrovascular accident caused by the reduction or interruption of local blood supply to brain tissue $^{[3]}$. Ischemic tissue will appear the corresponding regional neurological deficit symptoms, causing a series of complex biochemical molecular cascade reactions ${ }^{[4]}$, such as energy metabolism disorders, activation of excitotoxic glutamate signaling pathway, free radical response, inflammatory response and so on, usually to restore perfusion and reoxygenation for the treatment clinically. However, perfusion and reoxygenation will cause cerebral ischemia-reperfusion injury $(\mathrm{CIRI})^{[5]}$, a kind of brain tissue injury and related dysfunction caused by reperfusion of blood flow after cerebral ischemia, with the characteristics of high incidence, high disability rate and high mortality. Several mechanisms such as excitotoxicity, oxidative stress induced damage, apoptosis and inflammation are involved in the process of ischemiareperfusion ${ }^{[6,7]}$. Studies have shown that inflammation/immune response plays a key role in the pathogenesis of CIRI and has a dual role in promoting tissue damage and repair. Our previous studies have found that inflammation and cyclooxygenase-2 (COX-2) and its downstream pathways are involved in the mechanism of $\mathrm{CIR}{ }^{[8]}$, but whether the pathogenic mechanism of hydroxysafflor yellow $A(\mathrm{HYA})$ on CIRI involves COX-2/PGD2/DPs pathway has not been reported.

Safflower is a Chinese medicine that can promote blood circulation and remove blood stasis, which contains a variety of chemical components. Of safflower yellow pigment, HYA is the highest in content, and has the strongest pharmacological effect. Many research teams have indicated that HYA contains 
antioxidant ${ }^{[9]}$, anti-tumor ${ }^{[10]}$, and anti-ischemia reperfusion injury and other effects ${ }^{[11]}$. And HYA is widely used in the clinical treatment of dysemia diseases such as myocardial ischemia, cerebral ischemia, coronary heart disease, and cerebral thrombosis ${ }^{[12]}$. And there is no doubt that HYA is a promising lead drug candidate in designing new multi-targeted therapeutic agents against cardio-cerebrovascular diseases. So far, only the injection of HYA has been widely used in clinical therapy ${ }^{[13,14]}$.

However, its application was restricted by short half-life and low brain concentration. HYA is a hydrophilic compound with a chalcone glycoside structure, which has poor lipid solubility and low oral bioavailability ${ }^{[15]}$. So it is difficult for HYA to cross the BBB and reach the focus, and the BBB is still the main barrier for brain disease intervention ${ }^{[16]}$. In order to achieve the purpose of improving the oral absorption and bioavailability of HYA, HYA has been prepared into solid lipid nanoparticles ${ }^{[17]}$, a selfdouble-emulsifying drug delivery system ${ }^{[18]}$, water-in oil microemulsion ${ }^{[15]}$ and other dosage forms. All can improve the oral absorption of HYA, but the shortcomings of low bioavailability was not significantly improved, and when used for the treatment of brain diseases, the intracranial drug concentration is still insufficient. Therefore, the oral preparation of HYA for brain disease intervention is urgently needed.

Nanoemulsions are biphasic dispersion of two immiscible liquids: either the water in oil (W/O) or the oil in water $(\mathrm{O} / \mathrm{W})$ is stabilized by an amphiphilic surfactant ${ }^{[19]}$. Nanoemulsions consist of nano-scale droplets, which can slowly release, target accurately and of low toxicity. It is able to increase the contact area with the gastric mucosa, enhance the ability to absorb, get through the BBB, and prevent the hydrolysis of the wrapped substance or the degradation of enzyme ${ }^{[20,21]}$. It is a promising drug carrier in brain-targeted preparations ${ }^{[22,23]}$. Desai et al prepared oral Darunavir loaded lipid nanoemulsions, which improved the bioavailability of Darunavir and enhanced brain to absorb ${ }^{[20]}$. Phospholipids are amphiphilic, with good solubility in fat and biofilm compatibility. Drugs can be combined with phospholipids to prepare phospholipid complexes, thereby improving their bioavailability ${ }^{[24,25]}$. Results by Liu et al showed that the stability constant of the complex formed with HPCD was enhanced after flavanones are complexed with cyclodextrin $^{[26]}$. Chitosan is a cationic polysaccharide with the function of adhering mucous membrane, which can reversibly open Caco-2 cells to promote drug absorption ${ }^{[27]}$, Ma et al prepared the HYA-CS complex, the bioavailability of HYA was increased by 4.76 times $^{[28]}$. Lv et al also found that HYA is a Pglycoprotein substrate analog, which is easily excreted by P-glycoprotein in the intestinal tract, and leads to a strong bile efflux effect and low absorption ${ }^{[18]}$.

The effect of compound formulations was superior to that of single formulations, so it combined the advantages of multiple carriers to deliver HYA. Therefore, we formed HYA complexing with phospholipid and HPCD under certain conditions, and then prepared the water-in-oil HYA complex nanoemulsion with hyaluronic acid-modified multi-walled carbon nanotubes and chitosan (HC@HMC) by titration, hoping to improve the oral bioavailability and the ability to cross the BBB of HYA for treatment of ischemic stroke via oral administration. We measured the intestinal absorption and pharmacokinetics of HC@HMC in rats. And we determined the intracerebral concentration of HC@HMC after oral administration. Finally, we 
explored the protective effect of HC@HMC on cerebral ischemia-reperfusion in mice and HC@HMC may protect mice against cerebral ischemia-reperfusion injury through COX2/PGD2/DPs pathway.

\section{Materials And Methods}

\section{Materials}

Hydroxysafflor yellow A (HYA) (Purity 98\%) was purchased from Zhejiang Yongning Pharmaceutical Co., Ltd. (Taizhou, China), Rutin from Chengdu Munster Biotechnology Co., Ltd. (Chengdu, China), Hydroxypropyl- $\beta$-cyclodextrin from Jiangsu Taixing Xinxin Pharmaceutical Accessories Co., Ltd. (Taixing, China), Phospholipids from Shanghai Liberty Biotechnology Co., Ltd. (Shanghai China), Chitosan from Zhejiang Jinhuo Biochemical Co., Ltd. (Wenzhou, China), Glycerol monocaprylate (GMC) from Henan Zhengtong Food Technology Co., Ltd. (Xingyang China), Polyethylene glycol 400 from Sinopharm Group Chemical Reagent Co., Ltd. (Shanghai, China)and Polyoxyethylene hydrogenated castor oil (RH40) from Nanjing Durai Biotechnology Co., Ltd. (Nanjing China). Poloxamer 188 and 407 were obtained from Nanjing Well Biochemical Co. Ltd. (Nanjing, China). Hyaluronic acid modified multi-walled carbon nanotubes were made in our own laboratory. Methanol, acetonitrile (chromatographically pure) were purchased from America TEDIA Co., Ltd. (Ohio, America), and the remaining reagents were analytically pure.

\section{HPLC Analysis of HYA in vivo}

Chromatographic analysis was performed by using HPLC, the LC-20A HPLC system and UV-VIS 3150 ultraviolet-visible spectrophotometer (Shimadzu Corporation of Japan), a Phenomenex C18 (250 $\mathrm{mm} \times 4.6 \mathrm{~mm}, 5 \mu \mathrm{m})$ column. The detection wavelength was $403 \mathrm{~nm}$. Acetonitrile (A)-20 mmol. $\mathrm{L}^{-1}$ potassium dihydrogen phosphate solution $(B, p H=3.5)$, gradient elution for plasma sample and intestinal perfusion samples detection. The column oven temperature was $30^{\circ} \mathrm{C}$ and flow rate was $1 \mathrm{ml} \cdot \mathrm{min}^{-1}$. Rutin was used according to an internal standard.

\section{Synthesis of MWCNT-HA}

Firstly, synthesis carboxylated multi-walled carbon nanotubes (MWCNT-COOH), the mixed acid solution (the volume ratio of concentrated sulfuric acid and concentrated nitric acid is $3: 1$ ) and $30 \% \mathrm{H}_{2} \mathrm{O}_{2}$ solution $(10 \mathrm{~mL})$ were added into the MWCNT $(100 \mathrm{mg})$. After 3-hour ultrasonic reaction, it was diluted with a large amount of distilled water, using $0.22 \mu \mathrm{m}$ microporous filter membrane (mixed cellulose grease) to suck and filter, and then washed with distilled water to neutral, then put the filtered solid into the oven at a constant temperature, $80^{\circ} \mathrm{C}$ for drying, that was MWCNT-COOH.

Secondly, synthesis of aminated $\mathrm{HA}-\mathrm{NH} 2(\mathrm{HA}), \mathrm{HA}(200 \mathrm{mg})$ was dissolved in formamide $(10 \mathrm{~mL})$ at $50^{\circ} \mathrm{C}$, EDC (520 mg) and NHS (310 mg) dissolving in $10 \mathrm{~mL}$ and $5 \mathrm{~mL}$ formamide respectively, and the activation reaction was performed for $30 \mathrm{~min} .2 \mathrm{~mL}$ of ethylenediamine was dissolved in $10 \mathrm{~mL}$ of formamide. Under ice bath conditions, the activated HA solution was slowly dropped into the 
ethylenediamine solution, controlling the dripping rate (after $60 \mathrm{~min}$ of dripping). The reaction solution was warmed to room temperature, and reaction was continued for $3 \mathrm{~h}$. Then added a large amount of prechilled acetone precipitate, the precipitate was reconstituted with water, with a $0.45 \mu \mathrm{m}$ filter membrane, transfer to a dialysis bag $(\mathrm{MWCO}=3500)$ for $48 \mathrm{~h}$, and change the water every $6 \mathrm{~h}$, freeze-dried to get HA$\mathrm{NH} 2$.

Finally, MWCNT-COOH is combined with HA (MWCNT-HA), The amidation reaction of HA-NH2 and MWCNT-COOH under the conditions of EDC and NHS. Briefly, formamide $(30 \mathrm{~mL})$ was added into MWCNT-COOH (80 mg), transferred to a round-bottom flask after 30 minutes of sonication under ice bath conditions, rinsed with formamide $(20 \mathrm{~mL})$, and the formamide was transferred to a round bottom flask. EDC (305 mg) and NHS (182 mg) were dissolved in $5 \mathrm{~mL}$ formamide, and then transferred to a flask, activated at room temperature for $30 \mathrm{~min}$. Then, $10 \mathrm{~mL}$ formamide with HA-NH2 (160 mg) and triethylamine $(180 \mu \mathrm{L})$ was quickly added dropwise to the activated MWCNT-COOH, and reacted for $24 \mathrm{~h}$. Pre-cooled excess acetone (4 times of the reaction solution) was added in an ice bath, cooled, crystallized, and left to precipitate MWCNT-HA, then suck and filter with $0.22 \mu \mathrm{m}$ organic membrane, wash the precipitate with acetone, reconstitute with ultrapure water, using a dialysis bag (MWCO $=12000 \mathrm{kDa}$ ) to dialyze in water, and after $48 \mathrm{~h}$ freeze-drying to obtain the product MWCNT-HA.

\section{Preparation of HC@HMC}

HYA, HPCD and PC (molar ratio 1: 2.33: 2.47) are dissolved in a clean and dry round-bottom flask in an appropriate amount of absolute ethanol and stirred for $3.5 \mathrm{~h}\left(50^{\circ} \mathrm{C}\right)$ in the dark. Rotary evaporation removing the absolute ethanol to obtain HYA compound hydroxysafflor yellow A complex (HYAC); After HYAC was completely dissolved in the mixed solution of GMC, RH40, and PEG400, the chitosan solution $\left(2 \mathrm{mg} \cdot \mathrm{mL}^{-1}\right)$ was added dropwise. The solution will undergo a process from turbid to clear and transparent. Finally, a certain amount of novel hyaluronic acid-modified multi-walled carbon nanotubes (HMC), F188 and F407 mixture was added, and stirring was continued until the dissolution was complete, forming a clear, uniformly dispersed viscosity thick liquid, that was hydroxysafflor yellow Ahydroxypropyl- $\beta$-cyclodextrin phospholipid complex water-in-oil nanoemulsion with hyaluronic acidmodified multi-walled carbon nanotubes and chitosan modified (HC@HMC).

\section{Characterization of HC@HMC}

Malvern laser particle size potentiometer (Zetasizer Nano zs90; UK) was used to measure the particle size and Zeta potential of HC@HMC; conductivity meter (DDS-307A; Shanghai, China) was used to measure conductivity and the pH of HC@HMC.

\subsection{Absorption in the Intestine}

The in situ absorption of HYA or HC@HMC in the intestinal system of rats was investigated by the unidirectional perfusion method ${ }^{[29]}$. All rats were raised under controlled conditions and fasted more than $12 \mathrm{~h}$ before drug was administrated. Rats were anesthetized by intraperitoneal injection of $20 \%$ uratan solution $\left(7 \mathrm{~mL} \cdot \mathrm{kg}^{-1}\right)$. Then the rats were $\square x e d$ and a midline abdominal incision was made, found the 
duodenum, jejunum, ileum and colon, respectively, cut a small cannula at the upper end of the four intestine segments, ligated and fixed at the lower end, the wound covered with clean gauze soaked with saline. The contents were rinsed with physiological saline at a constant temperature $\left(37^{\circ} \mathrm{C}\right)$ and drained, then equilibrated with Krebs-Ringer solution at a flow rate of $0.25 \mathrm{~mL} \cdot \mathrm{min}^{-1}$ for $15 \mathrm{~min}$. Finally, HYA circulating liquid or $\mathrm{HC} @ H M C$ circulating liquid (the circulating liquid was placed in a $100 \mathrm{r} \cdot \mathrm{min}^{-1}$ magnetic stirrer) was injected and stirred at a flow rate of $0.25 \mathrm{~mL} \cdot \mathrm{min}^{-1}$. After $1 \mathrm{~h}$, we respectively collected the perfusate outflow at the outlets of the four intestinal segments. After the experiment, the four intestine segments were cut out, and the length $(L)$ and radius $(R)$ of each intestine segment were measured and recorded. The samples were sonicated with methanol for $20 \mathrm{~min}$, and the continuous filtrate filtered by $0.45 \mu \mathrm{m}$ microporous membrane was collected. For the $100 \mu \mathrm{L}$ intestinal juice sample, $10 \mu \mathrm{L} 50 \mu \mathrm{g} \cdot \mathrm{mL}^{-1} \mathrm{RT}$ and $50 \mu \mathrm{L}$ perchloric acid solution $\left(1 \mathrm{~mol} \cdot \mathrm{L}^{-1}\right)$ were added. After vortexing, centrifugation at $12000 \mathrm{rpm}$ for $10 \mathrm{~min}$, the supernatant was analyzed by HPLC.

The measured HYA content in the intestinal perfusion sample was substituted into the following three formulas to calculate the absorption rate constant (Ka), effective permeability coefficient (Peff) and absorption percentage (PA) of HYA and HC@HMC respectively.

$$
\begin{aligned}
& K_{a}=\frac{X_{0}-X_{t}}{C_{0} t \Pi R^{2} L}\left(\mathrm{E}-05,1 \cdot \mathrm{s}^{-1}\right) \\
& P_{\text {eff }} \frac{Q \times L_{n}\left(\frac{X_{\text {in }}}{X \text { out }}\right)}{2 \Pi P L}\left(\mathrm{E}-05, \mathrm{~cm} \cdot \mathrm{s}^{-1}\right) \\
& P A=\frac{X_{0}-X_{t}}{X_{0}}(\%)
\end{aligned}
$$

In this formula $X_{0}$ represents the total mass of the initial drug; $X_{t}$ represents the total mass of the drug at time $t ; C_{0}$ represents the initial drug concentration; $X_{\text {in }}$ represents the total mass of the drug entering the perfusate; $X_{\text {out }}$ represents the total mass of the drug flowing out of the perfusate; $t$ represents perfusion time; $Q$ stands for flow velocity; $R$ and $L$ are the inner diameter and length of each intestinal segment of perfusion. For the $K_{\mathrm{a}}, P_{\text {eff }}$ and $P A$, non-parametric statistical Wilcoxon signed rank sum test was used to compare the significant difference between HYA and HC@HMC $(P<0.05)$.

\section{Pharmacokinetic of HC@HMC}

Twelve male SD rats weighing $(230 \pm 20) \mathrm{g}$ were randomly divided into 2 groups and fasted for $12 \mathrm{~h}$, gavaged with HYA solution or HC@HMC (equivalent to HYA $6 \mathrm{mg} \cdot \mathrm{kg}^{-1}$ ). After administration, $0.5 \mathrm{~mL}$ blood was collected from the orbit of rats at different time points. Each sample was immediately transferred to heparin-infiltrated centrifuge tubes, after centrifugation at $6000 \mathrm{r} \cdot \mathrm{min}^{-1}$ for $10 \mathrm{~min}$, the supernatant $100 \mu \mathrm{L}$ was taken and added $10 \mu \mathrm{L} 50 \mu \mathrm{g} \cdot \mathrm{mL}^{-1} \mathrm{RT}$ and $50 \mu \mathrm{L} 6 \%$ perchloric acid solution into, then after vortexed and centrifuged at $12000 \mathrm{r} \cdot \mathrm{min}^{-1}$ for $10 \mathrm{~min}$, and the supernatant was measured by HPLC. Pharmacokinetic parameters were analyzed by DAS 2.1.1 using non-compartmental analysis, then analysis of variance and double one-sided t-test were carried out. 


\section{LC/MS Analysis of HYA in Brain}

Eighteen mice were randomly divided into six groups. After intravenous administration of HYA solution $\left(40 \mathrm{mg} \cdot \mathrm{kg}^{-1}\right)$ or oral administration of HC@HMC $\left(160 \mathrm{mg} \cdot \mathrm{kg}^{-1}\right)$, the mice were sacrificed $0.5 \mathrm{~h}, 1 \mathrm{~h}$ or 1.5 $\mathrm{h}$ later, and brain tissues were taken and weighed. $500 \mu \mathrm{L}$ of ultrapure water was added into the homogenizer and homogenized the brain tissue; centrifuged the homogenate $\left(12000 \mathrm{r} \cdot \mathrm{min}^{-1}, 10 \mathrm{~min}\right.$, $4^{\circ} \mathrm{C}$ ); then $100 \mu \mathrm{L}$ of supernatant was taken, $10 \mu \mathrm{L}$ of internal standard (RT) solution and $490 \mu \mathrm{L}$ of methanol were added into, centrifuged in the same way after mixing, the supernatant was used for sample injection.

Ultra High Liquid Chromatography Tandem Mass Spectrometry (Agilent 6470, Agilent Technologies, California, USA) used to detect intracranial HYA concentration in mice after administration, ACE Excel 2 C18-PFP column $(2.1 \times 100 \mathrm{~mm}, 2.0 \mu \mathrm{m})$ for separation, $0.1 \%$ formic acid-methanol was used as the mobile phase for gradient elution, the flow rate was $0.2 \mathrm{~mL} \cdot \mathrm{min}^{-1}$, and the injection volume was $5 \mu \mathrm{L}$. Use ESI+Agilent Jet Stream ion source, multi-reaction monitoring mode (MRM) detection. The internal standard method was used for quantification, rutin was used as the internal standard.

\section{Effect of HC@HMC on Cerebral Injury Caused by MCAO/R in Mice. Animals}

C57BL/ 6 male mice of 8-10 weeks (18 to $22 \mathrm{~g}$ ) old were purchased from the Laboratory Animal Center, Chongqing Medical University, China (license number: SYXK YU 2012-0001). The mice were housed under a temperature-controlled environment $\left(22-26^{\circ} \mathrm{C}\right)$ and humidity $(40-70 \%)$ with a $12 \mathrm{~h}$ light-dark cycle. The mice were supplied with standard rodent chow and water. All experiments in this study were consistent with the National Institute of Health Guide for the Care and Use of Laboratory Animals.

Middle cerebral artery occlusion/reperfusion model

Ischemic stroke was produced by using the MCAO/R method as previously reported ${ }^{[30]}$. The mice were anesthetized by intraperitoneal injection of sodium pentobarbital $\left(40 \mathrm{mg} \cdot \mathrm{kg}^{-1}\right)$. The right common carotid artery (CCA), external carotid artery (ECA), and the internal carotid artery (ICA) were exposed under a surgical microscope. The whole of the ECA and CCA were ligated. A nylon suture (Jialing, Guangzhou, China) was inserted from the CCA into the right side of ICA in a depth of $10 \pm 0.5 \mathrm{~mm}$, to occlude the origin of the middle cerebral artery (MCA). After 1-hour occlusion, reperfusion was achieved by withdrawing the suture to restore blood supply to the MCA territory. The sham-operated mice underwent the same surgery, but without the suture inserted. Body temperature was maintained $37 \pm 0.5^{\circ} \mathrm{C}$ during the surgery by a heating blanket.

\section{Protocol}

Mice were randomly divided into seven groups: sham group $(n=10), M C A O / R \operatorname{group}(n=10)$, MCAO/R+vehicle group ( $\mathrm{n}=10), \mathrm{MCAO} / \mathrm{R}+40 \mathrm{mg} \cdot \mathrm{kg}^{-1} \mathrm{HC} @ \mathrm{HMC}$ group $(\mathrm{n}=10), \mathrm{MCAO} / \mathrm{R}+80 \mathrm{mg} \cdot \mathrm{kg}^{-1}$ 
HC@HMC group ( $\mathrm{n}=10), \mathrm{MCAO} / \mathrm{R}+160 \mathrm{mg} \cdot \mathrm{kg}^{-1} \mathrm{HC} @ H M C$ group $(\mathrm{n}=10), \mathrm{MCAO} / \mathrm{R}+20 \mathrm{mg} \cdot \mathrm{kg}^{-1} \mathrm{HYA}$ group $(n=10)$. Mice were given HYA (Intravenous administration) and HC@HMC (Intragastric administration) 5 days before surgery, once a day. The brains of mice were removed at $24 \mathrm{~h}$ after MCAO/R operation. The recommended clinical dose of HYA is $100 \mathrm{mg}$ (approximately $2 \mathrm{mg} \cdot \mathrm{kg}^{-1}$ ) once a day for common cases or twice a day for severe cardiac patients ${ }^{[31]}$. Therefore, to mimic the current clinical treatment regimen, we chose to treat the ischemic mice with HYA at a dose of $20 \mathrm{mg} \cdot \mathrm{kg}^{-1}$. According to the absolute bioavailability, the dose of HC@HMC was $80 \mathrm{mg} \cdot \mathrm{kg}^{-1}$.

Neurological Scoring

The neurological deficit evaluation was conducted after 24h-reperfusion according to the Zea Longa' method as previously described (Longa et al. 1989) ${ }^{[32]}$. The criteria were used as follows: 0 , normal, no neurological deficit; 1 , mild neurological deficit, failure to completely extend the right forelimb; 2 , moderate neurological deficit, twisting to the contralateral side; 3 , severe neurological deficit, falling to the left; 4 , no ability of spontaneous motor activity. Animals that died during the process were not included in the statistics.

\section{Rota Rod Test}

Motor coordination was tested by using rota-rod tread-mill test ${ }^{[33]}$. The rota rod apparatus (UGO BASILE S.R.L, Italy) consisted of a rotating rod ( $75 \mathrm{~mm}$ diameter), on which the mice were allowed to hold. After twice daily training for 2 days (at a speed of $10 \mathrm{rpm}$ to $40 \mathrm{rpm}$ in 5 minutes), mice were tested 2 times as preoperative value on the third day. After 24 hours' reperfusion, mice were tested 2 times as postoperative value. The time for each mouse to remain on the rotating rod was recorded. The maximum time allowed was 5 minutes. The apparatus automatically records the time of falling.

\section{2,3,5-Triphenyltetrazolium Chloride Staining}

$24 \mathrm{~h}$ after reperfusion, the mice in all groups were decapitated after anesthesia, brains being removed and frozen in the refrigerator 20 minutes. Brains were cut into coronal sections of 2-mm thickness. The slices were stained with $2 \%(\mathrm{w} / \mathrm{v})$ 2,3,5-triphenyltetrazolium chloride (TTC)(sigma, USA) solution at $37^{\circ} \mathrm{C}$ in dark and then fixed in $4 \%(\mathrm{v} / \mathrm{v})$ phosphate-buffered paraformaldehyde solution. The infract areas appeared as a pale staining, which were measured on the posterior surface of each section by Image $\mathrm{J}$ software $(\mathrm{NIH}$, USA).

\section{Hematoxylin/eosin (HE) Staining}

Hematoxylin and eosin (HE) staining was used to observe pathological histological damage in the cerebral cortex and hippocampus. After 24-h reperfusion, the mice were anesthetized with sodium pentobarbital and perfused with PBS, and then perfused with $4 \%$ paraformaldehyde. After that, the brains were dehydrated by graded ethanol, embedded in paraffin, and cut into 5- $\mu \mathrm{m}$-thick sections. Finally, the sections were stained with HE reagents and observed by light microscopy. 


\section{Real-Time Polymerase Chain Reaction}

Total RNA was isolated from the mice cerebral cortex by Trizol reagent (Vazyme, Nanjing, China) according to the manufacturer's protocol. Reverse transcription of mRNA was performed using HiScript Q Select RT SuperMix (Vazyme, Nanjing, China). To detect the amount of COX2 mRNA and DP1 mRNA and DP2 mRNA. SYBR Green II (Biomake, USA) incorporation method was applied with ATGB being an internal control of mRNA. The primer sequences are reported in Table 1.

Table 1

List of primers used in QPCR analysis.

\begin{tabular}{|lll|}
\hline GENE & FORWARD Sequence $\left(\mathbf{5}^{\prime}->\mathbf{3}^{\prime}\right)$ & REVERSE Sequence $\left(\mathbf{5}^{\prime}->\mathbf{3}^{\prime}\right)$ \\
\hline COX2 & TGGTCTGGTGCCTGGTCTGATG & GCGGTTCTGATACTGGAACTGCTG \\
\hline DP1 & CACCCGTCCACAGTCAACACAC & GCCATTCTTCTCGCCCTTCCTG \\
\hline DP2 & AAGCACACCGCAGAGAATTG & TGTCTCAAGCCTTTCCCATTTTT \\
\hline ACTB & GTGCTATGTTGCTCTAGACTTCG & ATGCCACAGGATTCCATACC \\
\hline
\end{tabular}

\section{Western Blotting}

Mouse cortical samples were lysed with RIPA containing 1\% phenylmethanesulfonyl fluoride (PMSF) (100 $\mathrm{mM}$ ). The sample lysis buffer was collected into an EP tube and centrifuged at $12,000 \mathrm{rpm}$ and at $4{ }^{\circ} \mathrm{C}$ for $15 \mathrm{~min}$. Then the precipitate was discarded and the protein concentration was determined using the BCA Protein Assay Kit (Beijing, China). We performed Western blotting as described previously. In short, proteins were separated and transferred onto a polyvinylidene fluoride (PVDF) membrane (Millipore, USA). The membrane was incubated with primary antibodies overnight at $4^{\circ} \mathrm{C}$. After washing, the membranes were incubated with horseradish peroxidase-conjugated secondary antibodies (Proteintech, Wuhan, China) for $1 \mathrm{~h}$ at room temperature. At last, the immunoblots were developed by ECL western blotting detection reagent (Millipore, USA). The primary antibodies against COX2 (dilution 1:1000, Abcam, USA) and DP1 (dilution 1:1000, Abcam, USA) and were purchased from Abcam. DP2 (dilution 1:500, Aiffinity, China) were purchased from Aiffinity. and Tublin (dilution 1:5000, Proteintech, China) were purchased from Proteintech.

\section{Enzyme-linked Immunosorbent Assay}

The levels of PGD2, IL-1囚, and TNF-a were detected by following the protocol of a commercial ELISA kit (MeiMian, Jiangsu, China). Briefly, the sample, the standard, and a HRP-labeled antibody were added to microwells coated with the antibody, incubated at $37^{\circ} \mathrm{C}$ and washed thoroughly. The color was developed with a TMB substrate, which was converted to blue by peroxidase catalysis and finally converted to yellow by that action of acid. The intensity of the color was positively correlated with the expression of the target protein in the sample. The optical density value was measured with a microplate reader at a wavelength of $450 \mathrm{~nm}$, and the sample concentration was calculated. 


\section{Statistical Evaluation}

All data were reported as mean \pm standard deviation. Statistical significance was determined by $t$ test to compare two groups or one-way analysis of variance (ANOVA) followed by Tukey's multiple comparison test for multiple comparisons. Statistical analysis was performed by GraphPad Prism 6 (GraphPad Software, USA). $P$ value of less than 0.05 was regarded as statistically significant.

\section{Results}

\section{Characterization of HC@HMC}

The average Zeta potential of HC@HMC was about $0 \mathrm{mV}$. It can be seen from the appearance (Graphical abstract) that HC@HMC is a clear and uniformly dispersed liquid, which can preliminarily explain the successful preparation of HC@HMC.

\section{HC@HMC Improved the Oral Absorption of HYA Intestinal Perfusion in Rats}

Compared with HYA, HC@HMC showed higher absorption in total-intestinal tracts (duodenum, jejunum, ileum and colon). HYA and HC@HMC are mainly absorbed in the duodenum and colon. The determined $\mathrm{Ka}$, Peff and absorption percentage (PA) values comparison between HYA and HC@HMC the same gastro-intestinal areas were further described as follows (Fig. 1A).

The duodenal Ka value of $\mathrm{HC} @ \mathrm{HMC}$ was the largest in each intestinal segment, and the colon $K_{\mathrm{a}}$ value of HYA was the largest. The duodenal and ileum $K_{\mathrm{a}}$ values of $\mathrm{HC} @ \mathrm{HMC}$ were $17.82 \times \times 10^{-5} \mathrm{~s}^{-1}$ and $7.18 \times 10^{-5} \mathrm{~s}^{-1}$, which were respectively 15.10 times and 10.99 times of free HYA. The colonic $P_{\text {eff }}$ values of HC@HMC and HYA were the largest in each intestine, respectively $57.28 \times 10^{-5} \mathrm{~cm} \cdot \mathrm{s}^{-1}$ and $1.93 \times 10^{-5}$ $\mathrm{cm} \cdot \mathrm{s}^{-1}$. The colonic $P_{\text {eff }}$ value of HC@HMC were about 29.68 times of free HYA. The duodenal PA value of HC@HMC was the largest in each intestinal segment, while the colon PA value of HYA was the largest. The PA of HC@HMC in duodenum and colon was $79.41 \%$ and $79.02 \%$, respectively, 48.71 and 11.19 times of HYA.

\section{Pharmacokinetic of HC@HMC}

The main pharmacokinetic parameters of the non-compartmental model are shown in the Fig. 1. It can be seen from Figure 1B that within 0-4h, the blood concentration of HC@HMC is significantly higher than that of HYA. Due to the low solubility and poor permeability of HYA, the maximum blood concentration $\left(C_{\text {max }}\right)$ of HYA is only $1.25 \pm 0.33 \mathrm{mg} \cdot \mathrm{L}^{-1}$, while the $C_{\text {max }}$ of HC@HMC is as high as $20.14 \pm 4.23 \mathrm{mg} \cdot \mathrm{L}^{-1}$, which is 16.11 times that of HYA. In addition, the $A U C_{0-72 h}, T_{\text {max }}$ and MRT of HC@HMC are higher than those of HYA, and all parameters except $T_{\max }$ and $M R T_{0-96 h}$ are significantly different. The $A U C_{0-96 h}$ and 
$C_{\text {max }}$ of HC@HMC are about 10 times and 16 times of HYA, respectively, indicating that HC@HMC can increase the absorption of HYA in rats. Besides, the Cl of HYA is more than double that of HC@HMC, indicating that $\mathrm{HC} @ \mathrm{HMC}$ can increase the residence time in rats to promote absorption. The pharmacokinetic parameters of HC@HMC calculated by the non-compartment model is superior to HYA (Fig. 1C). So, the pharmacokinetic behavior of HC@HMC is superior to HYA. HYA and HC@HMC were found to not be bio-equivalent (Tab. 2). The oral bio-availability of HC@HMC compared with HYA was $999.63 \%$.

Table 2

The bioequivalence comparison of HSYA and HC@HMC after oral administration of HYA and HC@HMC (at the identical dose of $60 \mathrm{mg} \cdot \mathrm{kg}-1$ of HYA, $n=6$ )

\begin{tabular}{|c|c|c|c|c|c|}
\hline $\begin{array}{l}\text { Formulation } 1 \\
\text { and } 2\end{array}$ & Parameters & $\begin{array}{l}\text { Confidential } \\
\text { interval }\end{array}$ & $\begin{array}{l}\text { Bioequivalence } \\
\text { standard }\end{array}$ & $\begin{array}{l}P \\
\text { value }\end{array}$ & Bioequivalence \\
\hline \multirow[t]{4}{*}{$\begin{array}{l}\text { HYA and } \\
\text { HC@HMC }\end{array}$} & $\begin{array}{l}\text { AUC }(0-96 \\
\text { h) }\end{array}$ & $706.8 \% \otimes 761.5 \%$ & $80 \% \otimes 125 \%$ & & No \\
\hline & $\operatorname{AUC}(0-\infty)$ & $731.5 \% \otimes 782.7 \%$ & $80 \% \bigotimes 125 \%$ & & No \\
\hline & Cmax & $1272.7 \% \varangle 2096.9 \%$ & $70 \% \otimes 143 \%$ & & No \\
\hline & Tmax & & & 0.05 & No \\
\hline
\end{tabular}

\section{Intracranial Concentration of HYA in Mice}

To study whether our preparation can penetrate the BBB, we used liquid chromatograph-mass spectrometer (LC/MS) to determine the intracranial drug concentration in mice after administration. The results show that both $\mathrm{HC} @ H M C$ and HYA can penetrate the BBB, and $\mathrm{HC} @ H M C$ penetrates the BBB more. $1 \mathrm{~h}$ after oral administration of HC@HMC, HC@HMC reached the highest concentration 23.46 $\mathrm{ng} \cdot \mathrm{mL}^{-1}$, which was 1.15 times that of HYA (Fig. 2). It is speculated that the water-in-oil nanoemulsion improves the liposolubility, so it penetrates more through the BBB. Combining the blood drug concentration curve and the intracranial drug concentration curve, the change trend and peak time of HC@HMC are almost the same (Fig. 1B). This result shows that HC@HMC improves the absorption of intestine first, and then more easily penetrates the BBB.

\section{Effect of HC@HMC on Cerebral Injury Caused by MCAO/R in Mice}

To determine whether HC@HMC had effects on CIRI, the TTC assay was used to detect the brain infarct size. Representative images of TTC-stained brain sections are shown in Fig. 3A. Obviously, compared with the sham group, the infarct volume was significantly increased in MCAO/R group. Compared with MCAO/R group, 80 mg $\cdot \mathrm{kg}^{-1} \mathrm{HC} @ H M C$ group and $160 \mathrm{mg} \cdot \mathrm{kg}^{-1} \mathrm{HC} @ H M C$ group and $20 \mathrm{mg} \cdot \mathrm{kg}^{-1} \mathrm{HYA}$ group significantly decreased the infarct volume and neurological score (Fig. 3B, 3C). Compared with the sham 
group, the residence time of mice on the rotating rod was significantly reduced. Compared with MCAO/R group, $80 \mathrm{mg} \cdot \mathrm{kg}^{-1} \mathrm{HC} @ H M C$ group and $160 \mathrm{mg} \cdot \mathrm{kg}^{-1} \mathrm{HC} @ H M C$ group and $20 \mathrm{mg} \cdot \mathrm{kg}^{-1} \mathrm{HYA}$ group significantly improved the motor ability and coordination ability of mice after surgery (Fig. 3D).

To determine whether the brain had histopathological changes, HE staining was conducted. In the sham group, nerve cells were arranged tightly in order, and most cells were round or oval with large cell bodies. However,in the MCAO/R group, vehicle group, and $40 \mathrm{mg} \cdot \mathrm{kg}^{-1} \mathrm{HC} @ \mathrm{HMC}$ group, nerve cells presented significant nuclear pyknosis, vacuolization and disordered arrangement (Fig. 4A,4C). Compared with the MCAO/R group, 80 mg $\cdot \mathrm{kg}^{-1} \mathrm{HC} @ H M C$ group and $160 \mathrm{mg} \cdot \mathrm{kg}^{-1} \mathrm{HC} @ \mathrm{HMC}$ group and $20 \mathrm{mg} \cdot \mathrm{kg}^{-1} \mathrm{HYA}$ group reduced the nuclear deep staining of the nucleus, the vacuolization and the mortality of nerve cells (Fig. 4B,4D).

\section{HC@HMC Reduces Inflammation in MCAO/R Treated-mice.}

We examine whether HC@HMC had effects on COX2, DP1, DP2 expression. Compared with the sham group, the expressions of COX2, DP1, DP2 mRNA and protein were significantly increased in the cortex of MCAO/R-treated mice. Compared with the model group, HC@HMC markedly decreased the expression of COX2, DP1, DP2 mRNA and protein in the cortex of MCAO/R-treated mice, and HYA had the same effect. (Fig. 5A,5B,5C,5D,5E,5F).

To determine whether related products and inflammatory factors had changed, ELISA assay was used to test PGD2, TNF-a, IL-1 $\beta$ content in the mouse cortex. Compared with the sham group, the mice cortical PGD2, TNF-a, IL-1 $\beta$ content were significantly increased in the model group. Compared with the model group, HC@HMC significantly blunted the increase of mice cortical PGD2, TNF- $\alpha$ and IL-1 $\beta$ content, and HYA had the same effect (Fig. 5H,5l,5J).

\section{Conclusion}

Generally speaking, drugs for the treatment of stroke could pass through the BBB to be meaningful. Studies have shown that the gastrointestinal absorption of HYA is poor, and oral bioavailability is low ${ }^{[15]}$. So the main application of HYA is intravenous administration. Therefore, there is an urgent need for powerful nanomaterials to overcome the problems of poor blood stability and low BBB penetration efficiency of HYA.

Here, we formed HYA complexing with phospholipid and HPCD under certain conditions, and then prepared the water-in-oil HYA complex nanoemulsion with hyaluronic acid-modified multi-walled carbon nanotubes and chitosan (HC@HMC) by titration. The results showed that HC@HMC significantly 
improved the absorption of HYA, thereby increasing its bioavailability, which may reduce its efflux by Pglycoprotein and improve the absorption of HYA. Because polyethylene glycol 400 acts as a Pglycoprotein (P-gp) inhibitor, which promotes the absorption of P-gp substrate HYA. We firstly prepared HYA with phospholipids and HPCD into a complex. The phospholipids could increase cell membrane fluidity, which may help open the tight junctions between cells ${ }^{[34,35]}$, resulting in the enhanced permeability of hydrophilic drugs. Chitosan can also enhance drug permeability across the BBB by affecting the tight junction ${ }^{[36]}$. HPCD can improve the stability of HYA and reduce the degradation of HYA ${ }^{[26]}$, improving its bioavailability and activity. $\mathrm{HC} @ \mathrm{HMC}$ can significantly increase the $C_{\max }$ of HYA and prolong $M R T$, thereby significantly increasing its $A U C$ and achieving the purpose of improving the bioavailability of HYA.

In addition to increasing drug plasma concentrations, effective BBB penetration is another major challenge in stroke treatment. Combined with LC/MS results, after oral administration of HC@HMC 1 h, the highest concentration of HC@HMC was $23.46 \mathrm{ng} / \mathrm{ml}$, which was 1.15 times of HYA. Therefore, oral administration of HC@HMC can achieve intravenous drug concentrations of HYA. We prepared HYA into oral preparations, which can improve the compliance of patients in clinical practice. The water-in-oil nanoemulsion is an effective strategy for oral delivery of highly water-soluble and low-permeability drugs, and an effective strategy of crossing the BBB.

Finally, we investigated the therapeutic effect of HC@HMC in MCAO/R-treated mice. Li sun et al found that injecting HYA $\left(1,5,10 \mathrm{mg} \cdot \mathrm{kg}^{-1}\right)$ into the tail vein 30 minutes before surgery protects rats from focal cerebral CIRI through inhibited I/R-induced protein oxidation and nitration ${ }^{[37]}$. In the present study, by using the middle cerebral artery occlusion (MCAO) model, Lu Yu et al found that $8 \mathrm{mg} \cdot \mathrm{kg}^{-1}$ and 16 $\mathrm{mg} \cdot \mathrm{kg}^{-1}$ HYA administration by common carotid artery (CCA) injection improved impaired cognitive function in Morris water maze(MWM) and passive avoidance tasks, suggesting that HYA treatment in a certain concentration can improve cognitive impairment in MCAO rats ${ }^{[38]}$. The recommended clinical dose of HYA is $100 \mathrm{mg}$ (approximately $2 \mathrm{mg} \cdot \mathrm{kg}^{-1}$ ) once a day for common cases or twice a day for severe cardiac patients $^{[31]}$. Therefore, to mimic the current clinical treatment regimen, we chose to treat the ischemic mice with HYA at a dose of $20 \mathrm{mg} \cdot \mathrm{kg}^{-1}$. According to our experiments, intravenous injection of HYA $6 \mathrm{mg} \cdot \mathrm{kg}^{-1} \mathrm{AUC}_{0-24 \mathrm{~h}}$ is $(23.85 \pm 0.21) \mathrm{mg} \cdot \mathrm{h} \cdot \mathrm{L}^{-1}$, oral HC@HMC $60 \mathrm{mg} \cdot \mathrm{kg}^{-1} \mathrm{AUC}_{0-24 \mathrm{~h}}$ is $(54.48 \pm 9.87)$ $\mathrm{mg} \cdot \mathrm{h} \cdot \mathrm{L}^{-1}$. The calculated absolute bioavailability of HC@HMC is $23 \%$, so the effective dose of HC@HMC is $87 \mathrm{mg} \cdot \mathrm{kg}^{-1}$. For ease of calculation, we finally set the dose to $80 \mathrm{mg} \cdot \mathrm{kg}^{-1}$. We also set a low dose of $40 \mathrm{mg} \cdot \mathrm{kg}^{-1}$ and a high dose of $160 \mathrm{mg} \cdot \mathrm{kg}^{-1}$. From the results, $80 \mathrm{mg} \cdot \mathrm{kg}^{-1} \mathrm{HC} @ \mathrm{HMC}$ and $160 \mathrm{mg} \cdot \mathrm{kg}^{-1}$ $\mathrm{HC} @ H M C$ and $20 \mathrm{mg} \cdot \mathrm{kg}^{-1} \mathrm{HYA}$ are all effective doses, and there was a certain dose-dependent. These results indicate that HC@HMC increase the bioavailability of HYA, thereby protecting mice from focal cerebral CIRI.

Prior to the study of mechanism, 3 doses (40,80 and $120 \mathrm{mg} \cdot \mathrm{kg}^{-1}$ ) of HC@HMC were assessed according to neurological function score. The low-dose group showed no improvement of the 
neurological function scores. There were obvious improvements of the function scores in the two highdose groups. However, there was no significant inter-group difference. So we choose the $80 \mathrm{mg} \cdot \mathrm{kg}^{-1}$ group to study the mechanism of HC@HMC. From our results, compared with the sham group, the expressions of COX2, DP1 and DP2 in the model group were significantly increased. Inflammation plays an important role in the occurrence and development of central nervous system diseases ${ }^{[39]}$. $\operatorname{coX} 2$ is a key rate-limiting enzyme in the inflammatory pathway ${ }^{[40]}$. PGD2 was downstream products of COX2, which was abundant prostaglandins in brain ${ }^{[41]}$. Studies have found that some harmful effects of PGD2 are mainly regulated by DP2 receptors or through the PPARY pathway ${ }^{[42]}$. Compared to the model group. $\mathrm{HC} @ H M C$ 80 $\mathrm{mg} \cdot \mathrm{kg}^{-1}$ group can significantly reduce the expression of COX2, DP1 and DP2. This is the first study that demonstrated the protective effect of HC@HMC on reducing ischemia-reperfusion cerebral injury through COX2/PGD2/DPs pathway. HYA has the same effect, which shows that HC@HMC and HYA have the same mechanism. However, it is not clear whether HYA exerts its protective effect through the drug substance or its metabolites, and further research is needed. Studies have found that HYA has 8 main metabolites in rats, but we do not know which one plays an important role ${ }^{[43]}$. I believe that there will be more high-quality studies to confirm it in the future, which will provide more possibilities for the treatment and prognosis of ischemic stroke risk groups.

In summary, we developed an effective strategy to survive HYA through the BBB with good stability, which ameliorated cerebral ischemia-reperfusion injury in mice. These results indicate that our HC@HMC nanomedicine has good clinical therapeutic potential for stroke owing to ease of formulation, stability and BBB permeability.

\section{Declarations}

\section{Ethics approval and consent to participate}

This experiment has been approved by the Ethics Committee of Chongqing Medical University. All experiments in this study were consistent with the National Institute of Health Guide for the Care and Use of Laboratory Animals.

\section{Consent for publication}

Not applicable.

\section{Availability of data and material}

All data generated or analysed during this study are included in this published article.

\section{Competing interests}

The authors declare that they have no competing interests.

\section{FUNDING}


This work was financially supported by the Chongqing Education Committee (CYS20212), Chongqing Science and Technology Committee (cstc2017shmsA130028), and China Postdoctoral Science Foundation (2019m663451).

\section{AUTHORS' CONTRIBUTIONS}

Qiong Wang and Cailing Zhong designed and completed most animal experiments. Jingqing Zhang, Hua Zhao and Yuru Huang designed and synthesized nanoemulsions of hydroxysafflor yellow A. Dezhang Zhao mainly completed the LC/MS related experiments. All authors have given approval to the final version of the manuscript.

\section{Acknowledgements}

We appreciated Zhe Peng for the experimental design of this manuscript.

\section{References}

1. Hankey, G. J. 2017. Stroke. Lancet 389: 641-654.

2. Mozaffarian, D., E. J. Benjamin, A. S. Go, D. K. Arnett, M. J. Blaha, and M. Cushman, et al. 2015. Heart disease and stroke statistics-2015 update: a report from the American Heart Association. Circulation 131: e29-e322.

3. Chen, L., G. Zhang, Y. Gu, and X. Guo. 2016. Meta-Analysis and Systematic Review of Neural Stem Cells therapy for experimental ischemia stroke in preclinical studies. Scientific reports 6: 32291.

4. Sommer, C. J. 2017. Ischemic stroke: experimental models and reality. Acta Neuropatho/133: 245261.

5. Yellon, D. M., and D. J. Hausenloy. 2007. Myocardial reperfusion injury. The New England journal of medicine 357: 1121-1135.

6. Hosoo, H., A. Marushima, Y. Nagasaki, A. Hirayama, H. Ito, and S. Puentes, et al. 2017. Neurovascular Unit Protection From Cerebral Ischemia-Reperfusion Injury by Radical-Containing Nanoparticles in Mice. Stroke 48: 2238-2247.

7. Mizuma, A., and M. A. Yenari. 2017. Anti-Inflammatory Targets for the Treatment of Reperfusion Injury in Stroke. Frontiers in neurology 8: 467.

8. Yu, L., B. Yang, J. Wang, L. Zhao, W. Luo, and Q. Jiang, et al. 2014. Time course change of COX2PGI2/TXA2 following global cerebral ischemia reperfusion injury in rat hippocampus. Behavioral and brain functions: BBF 10: 42.

9. Wang, Y., C. Zhang, W. Peng, Z. Xia, P. Gan, and W. Huang, et al. 2016. Hydroxysafflor yellow A exerts antioxidant effects in a rat model of traumatic brain injury. Molecular medicine reports 14: 36903696.

10. Yang, F., J. Li, J. Zhu, D. Wang, S. Chen, and X. Bai. 2015. Hydroxysafflor yellow A inhibits angiogenesis of hepatocellular carcinoma via blocking ERK/MAPK and NF-KB signaling pathway in 
H22 tumor-bearing mice. European journal of pharmacology 754: 105-114.

11. Lv, Y., Y. Qian, L. Fu, X. Chen, H. Zhong, and X. Wei. 2015. Hydroxysafflor yellow A exerts neuroprotective effects in cerebral ischemia reperfusion-injured mice by suppressing the innate immune TLR4-inducing pathway. Eur J Pharmacol 769: 324-332.

12. Liu, Y. N., Z. M. Zhou, and P. Chen. 2008. Evidence that hydroxysafflor yellow A protects the heart against ischaemia-reperfusion injury by inhibiting mitochondrial permeability transition pore opening. Clinical and experimental pharmacology \& physiology 35: 211-216.

13. Qin, F., and X. Huang. 2009. Guanxin II (II) for the management of coronary heart disease. Chinese journal of integrative medicine 15: 472-476.

14. Liu, S. X., Y. Zhang, Y. F. Wang, X. C. Li, M. X. Xiang, and C. Bian, et al. 2012. Upregulation of heme oxygenase-1 expression by hydroxysafflor yellow A conferring protection from anoxia/reoxygenationinduced apoptosis in H9c2 cardiomyocytes. International journal of cardiology 160: 95-101.

15. Qi, J., J. Zhuang, W. Wu, Y. Lu, Y. Song, and Z. Zhang, et al. 2011. Enhanced effect and mechanism of water-in-oil microemulsion as an oral delivery system of hydroxysafflor yellow A. International journal of nanomedicine 6: 985-991.

16. Liebner, S., R. M. Dijkhuizen, Y. Reiss, K. H. Plate, D. Agalliu, and G. Constantin. 2018. Functional morphology of the blood-brain barrier in health and disease. Acta Neuropathol 135: 311-336.

17. Zhao, B., S. Gu, Y. Du, M. Shen, X. Liu, and Y. Shen. 2018. Solid lipid nanoparticles as carriers for oral delivery of hydroxysafflor yellow A. International journal of pharmaceutics 535: 164-171.

18. Lv, L. Z., C. Q. Tong, Q. Lv, X. J. Tang, L. M. Li, and Q. X. Fang, et al. 2012. Enhanced absorption of hydroxysafflor yellow A using a self-double-emulsifying drug delivery system: in vitro and in vivo studies. International journal of nanomedicine 7: 4099-4107.

19. Singh, Y., J. G. Meher, K. Raval, F. A. Khan, M. Chaurasia, and N. K. Jain, et al. Nanoemulsion: Concepts, development and applications in drug delivery. Journal of controlled release: official journal of the Controlled Release Society 2017; 252: 28-49.

20. Desai, J., and H. Thakkar. 2019. Enhanced oral bioavailability and brain uptake of Darunavir using lipid nanoemulsion formulation. Colloids and surfaces. B, Biointerfaces 175: 143-149.

21. Karami, Z., M. R. Saghatchi Zanjani, and M. Hamidi. 2019. Nanoemulsions in CNS drug delivery: recent developments, impacts and challenges. Drug discovery today 24: 1104-1115.

22. Nirale, P., A. Paul, and K. S. Yadav. 2020. Nanoemulsions for targeting the neurodegenerative diseases: Alzheimer's, Parkinson's and Prion's. Life sciences 245: 117394.

23. Đorđević, S. M., A. Santrač, N. D. Cekić, B. D. Marković, B. Divović, and T. M. Ilić, et al. 2017. Parenteral nanoemulsions of risperidone for enhanced brain delivery in acute psychosis: Physicochemical and in vivo performances. International journal of pharmaceutics 533: 421-430.

24. Abd-Elsalam, W. H., S. N. El-Helaly, M. A. Ahmed, and A. M. Al-Mahallawi. 2018. Preparation of novel phospholipid-based sonocomplexes for improved intestinal permeability of rosuvastatin: In vitro characterization, dynamic simulation, Caco-2 cell line permeation and in vivo assessment studies. International journal of pharmaceutics 548: 375-384. 
25. Wu, H., X. Long, F. Yuan, L. Chen, S. Pan, and Y. Liu, et al. 2014. Combined use of phospholipid complexes and self-emulsifying microemulsions for improving the oral absorption of a BCS class IV compound, baicalin. Acta pharmaceutica Sinica. B 4: 217-226.

26. Liu, B., W. Li, T. A. Nguyen, and J. Zhao. 2012. Empirical, thermodynamic and quantum-chemical investigations of inclusion complexation between flavanones and (2-hydroxypropyl)-cyclodextrins. Food chemistry 134: 926-932.

27. Wang, Y., X. Bai, B. Hu, M. Xing, Q. Cao, and A. Ji, et al. Transport Mechanisms of Polymannuronic Acid and Polyguluronic Acid Across Caco-2 Cell Monolayers. Pharmaceutics 2020; 12.

28. Ma, G. N., F. L. Yu, S. Wang, Z. P. Li, X. Y. Xie, and X. G. Mei. 2015. A novel oral preparation of hydroxysafflor yellow $A$ base on a chitosan complex: a strategy to enhance the oral bioavailability. An Official Journal of the American Association of Pharmaceutical Scientists 16: 675-682.

29. Sun, L., K. Wan, X. Hu, Y. Zhang, Z. Yan, and J. Feng, et al. 2016. Functional nanoemulsion-hybrid lipid nanocarriers enhance the bioavailability and anti-cancer activity of lipophilic diferuloylmethane. Nanotechnology 27: 085102.

30. Yu, H., L. Song, X. Cao, W. Li, Y. Zhao, and J. Chen, et al. 2020. Hederagenin Attenuates Cerebral Ischaemia/Reperfusion Injury by Regulating MLK3 Signalling. Frontiers in pharmacology 11: 1173.

31. Qi, Z., F. Yan, W. Shi, C. Zhang, W. Dong, and Y. Zhao, et al. 2014. AKT-related autophagy contributes to the neuroprotective efficacy of hydroxysafflor yellow A against ischemic stroke in rats. Translational stroke research 5: 501-509.

32. Longa, E. Z., P. R. Weinstein, S. Carlson, and R. Cummins. 1989. Reversible middle cerebral artery occlusion without craniectomy in rats. Stroke 20: 84-91.

33. Doeppner, T. R., B. Kaltwasser, M. Bähr, and D. M. Hermann. 2014. Effects of neural progenitor cells on post-stroke neurological impairment-a detailed and comprehensive analysis of behavioral tests. Frontiers in cellular neuroscience 8: 338.

34. Sha, X., G. Yan, Y. Wu, J. Li, and X. Fang. 2005. Effect of self-microemulsifying drug delivery systems containing Labrasol on tight junctions in Caco-2 cells. European journal of pharmaceutical sciences: official journal of the European Federation for Pharmaceutical Sciences 24: 477-486.

35. Ujhelyi, Z., F. Fenyvesi, J. Váradi, P. Fehér, T. Kiss, and S. Veszelka, et al. 2012. Evaluation of cytotoxicity of surfactants used in self-micro emulsifying drug delivery systems and their effects on paracellular transport in Caco-2 cell monolayer. European journal of pharmaceutical sciences: official journal of the European Federation for Pharmaceutical Sciences 47: 564-573.

36. Yu, S., X. Xu, J. Feng, M. Liu, and K. Hu. 2019. Chitosan and chitosan coating nanoparticles for the treatment of brain disease. International journal of pharmaceutics 560: 282-293.

37. Sun, L., L. Yang, Y. W. Xu, H. Liang, J. Han, and R. J. Zhao, et al. 2012. Neuroprotection of hydroxysafflor yellow $A$ in the transient focal ischemia: inhibition of protein oxidation/nitration, 12/15-lipoxygenase and blood-brain barrier disruption. Brain research 1473: 227-235.

38. Yu, L., Y. Duan, Z. Zhao, W. He, M. Xia, and Q. Zhang, et al. 2018. Hydroxysafflor Yellow A (HSYA) Improves Learning and Memory in Cerebral Ischemia Reperfusion-Injured Rats via Recovering 
Synaptic Plasticity in the Hippocampus. Frontiers in cellular neuroscience 12: 371.

39. Serhan, A., J. L. Aerts, E. Boddeke, and R. Kooijman. 2020. Neuroprotection by Insulin-like Growth Factor-1 in Rats with Ischemic Stroke is Associated with Microglial Changes and a Reduction in Neuroinflammation. Neuroscience 426: 101-114.

40. Yang, Y., Q. Chen, Q. Zhao, Y. Luo, Y. Xu, and W. Du, et al. 2019. Inhibition of COX2/PGD2-Related Autophagy Is Involved in the Mechanism of Brain Injury in T2DM Rat. Frontiers in cellular neuroscience 13: 68.

41. Chiu, E. K., and J. S. Richardson. 1985. Behavioral and neurochemical aspects of prostaglandins in brain function. General pharmacology 16: 163-175.

42. Doré, S., and A. Shafique Ahmad. Frontiers in Neuroengineering Cytoprotective Role of Prostaglandin D(2) DP1 Receptor against Neuronal Injury Following Acute Excitotoxicity and Cerebral Ischemia. In: Kobeissy FH, Editor Brain Neurotrauma: Molecular, Neuropsychological, and Rehabilitation Aspects. (CRC Press/Taylor \& Francis @ 2015 by Taylor \& Francis Group, LLC., Boca Raton (FL), 2015).

43. Jin, Y., L. Wu, Y. Tang, Y. Cao, S. Li, and J. Shen, et al. 2016. UFLC-Q-TOF/MS based screening and identification of the metabolites in plasma, bile, urine and feces of normal and blood stasis rats after oral administration of hydroxysafflor yellow A. Journal of chromatography. B, Analytical technologies in the biomedical and life sciences 1012-1013: 124-129.

\section{Figures}



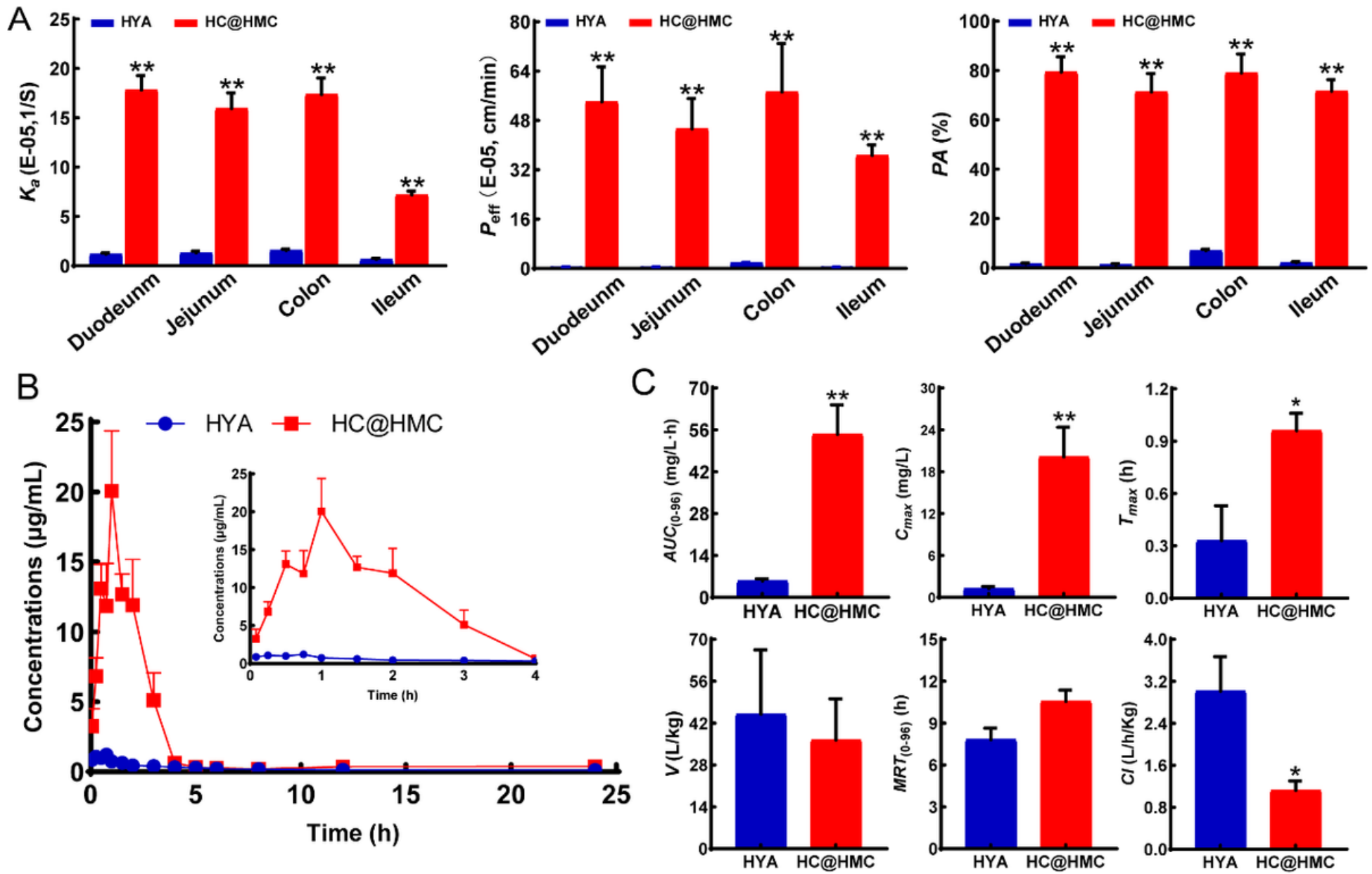

Figure 1

Intestinal absorption and in vivo kinetic characteristics of HYA and HC@HMC. (A) The main intestinal absorption parameters (Ka, Peff, PA) of HYA and HC@HMC in four intestinal segments. (B) Pharmacokinetics of HYA and HC@HMC (C) main pharmacokinetic parameters of HYA and HC@HMC showing kinetic characteristics. The data were shown as mean \pm standard deviation. $n=6,{ }^{*} P<0.05$ or **P<0.01 indicated significant differences or very significant differences between free HYA and HC@HMC. 


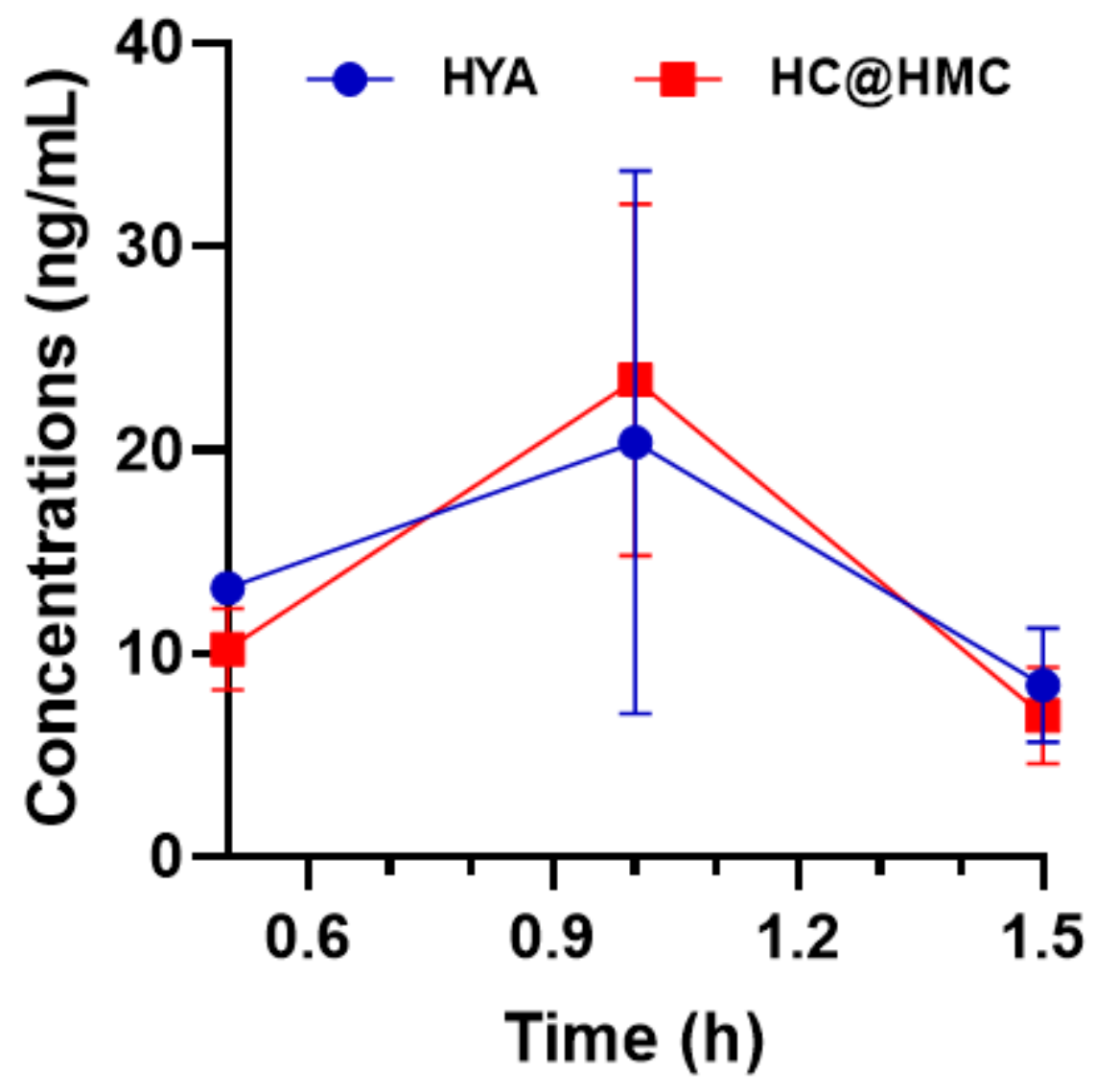

Figure 2

Intracranial concentration of HYA in mice. Line graph of drug concentration at $0.5 \mathrm{~h}, 1 \mathrm{~h}$, and $1.5 \mathrm{~h}$. Data are presented as the mean $\pm S D, n=3$. 

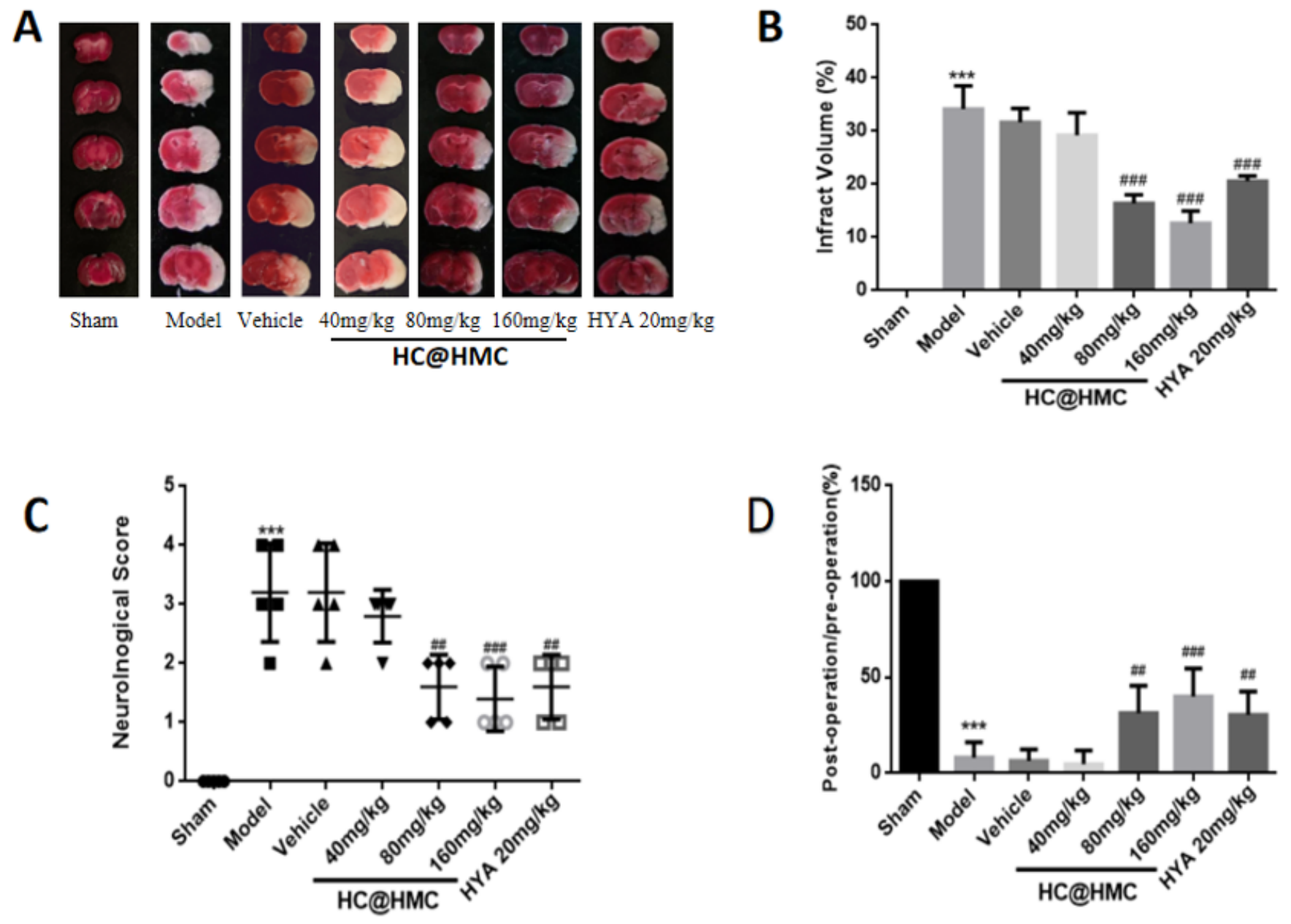

Figure 3

Effect of HC@HMC on brain injury in MCAO/R-treated mice. (A) Representative images of TTC staining of infarct size in brain sections; (B) Quantification of infarct volume by TTC staining after $24 \mathrm{~h}$ of reperfusion ( $n=3)$; (C) Neurological score were estimated after 24 h of reperfusion $(n=5)$; (D)Rota rod test were estimated after $24 \mathrm{~h}$ of reperfusion $(n=5)$. Data are presented as the mean $\pm S D$. ${ }^{*} P<0.05,{ }^{*} P<0.01$

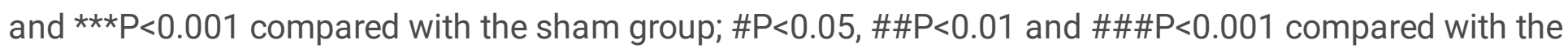
model group. 


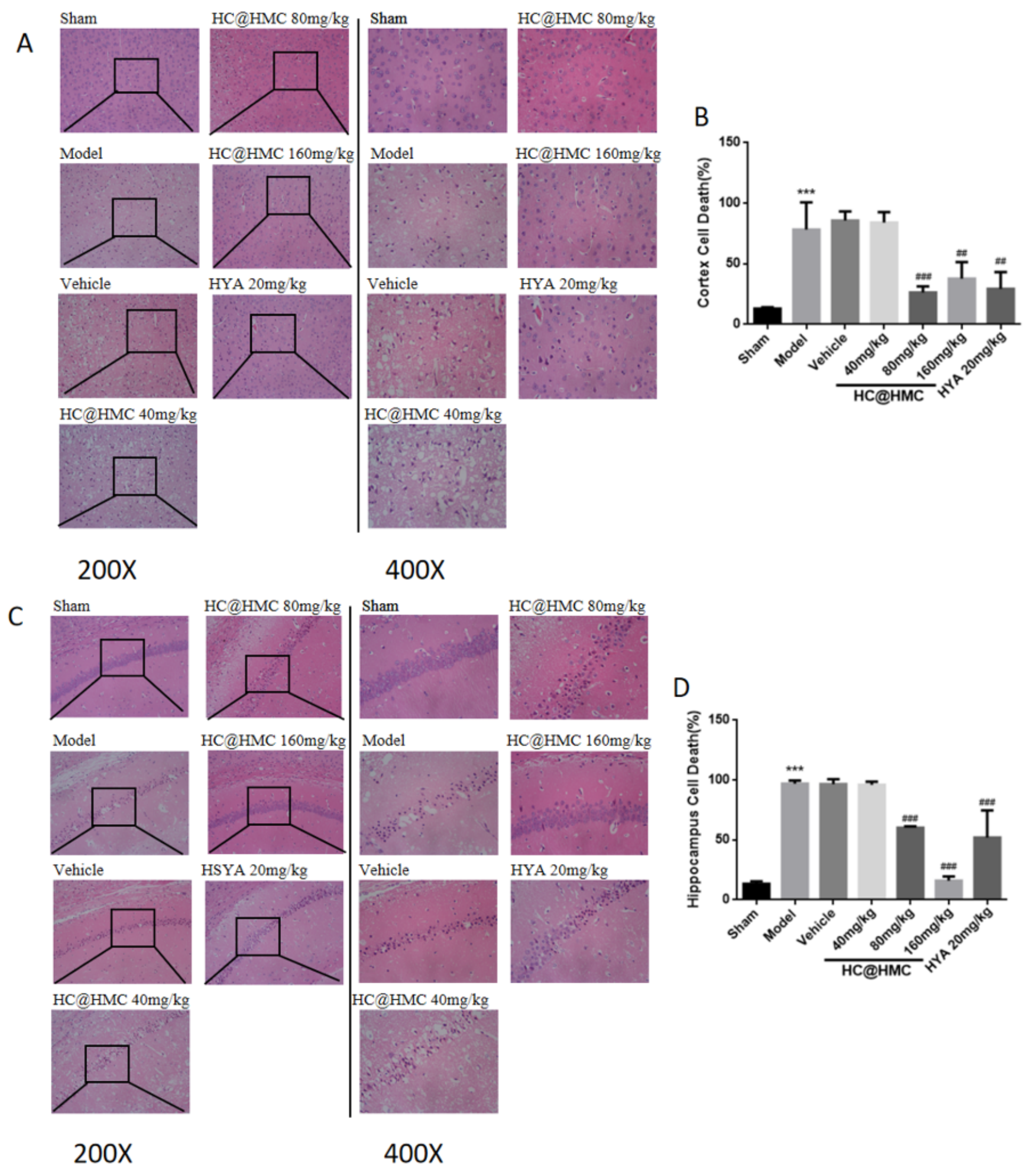

\section{Figure 4}

Histopathology of hippocampus and cortex in mice. (A, B) Representative images of histopathological assay of mice in cortex $(n=3$, scale bar $=50 \mu \mathrm{m}) ;(C, D)$ Representative images of histopathological assay of mice in hippocampus $(n=3$, scale bar $=50 \mu \mathrm{m})$. Data are presented as the mean $\pm S D$. ${ }^{*} P<0.05,{ }^{*} P<0.01$

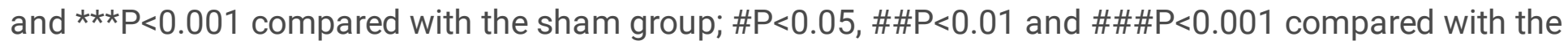
model group. 
A

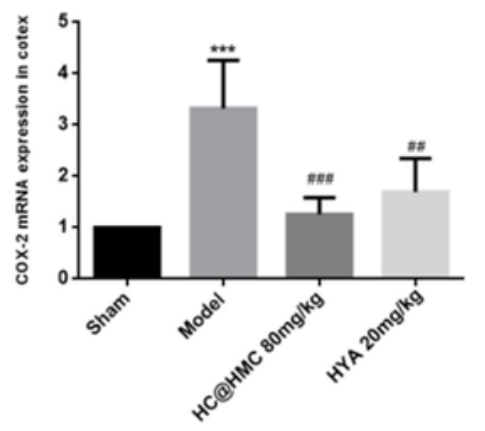

D

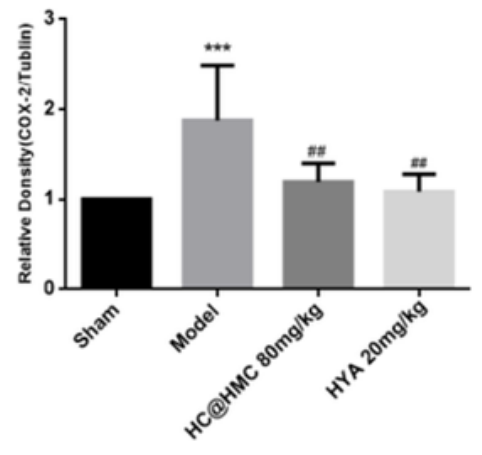

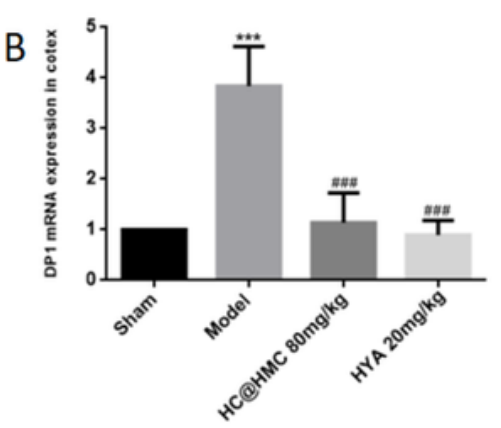

$E_{\text {를 }}$

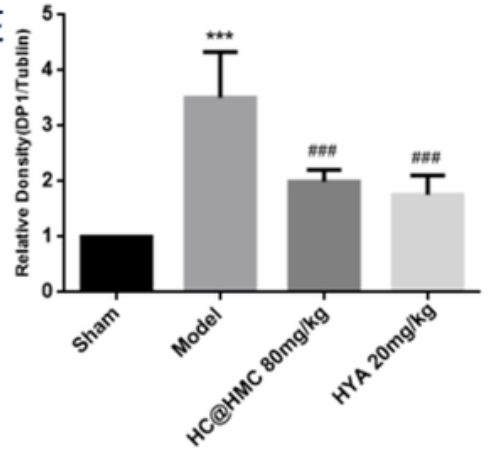

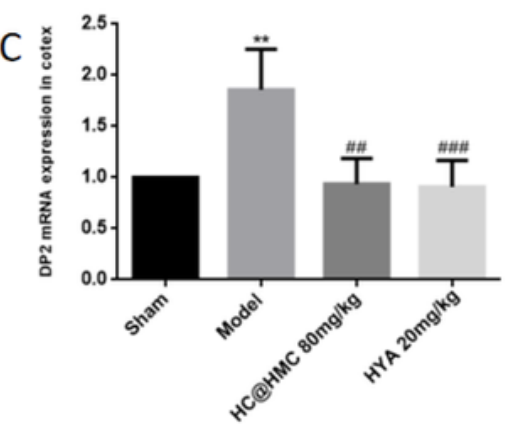

$\mathrm{F}$ 흫

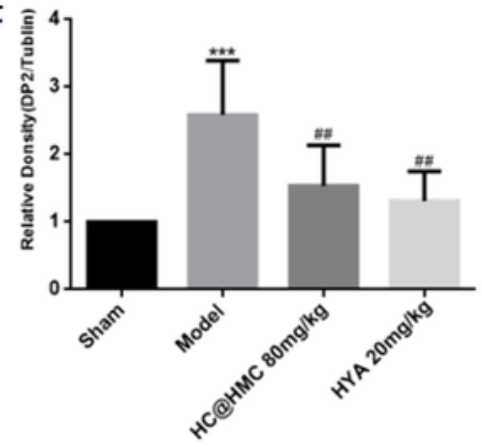

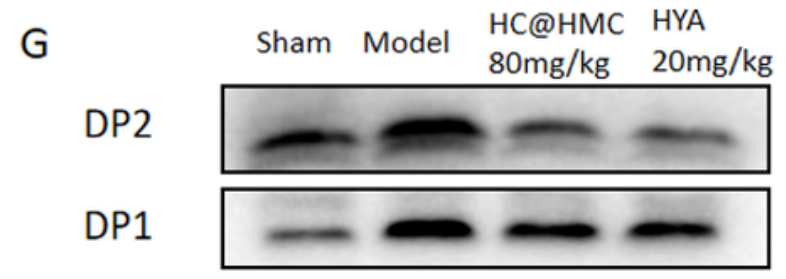

COX-2

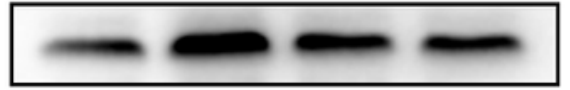

Tublin

$\mathrm{H}$

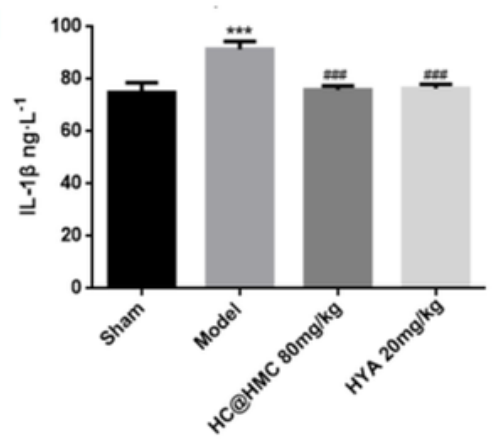

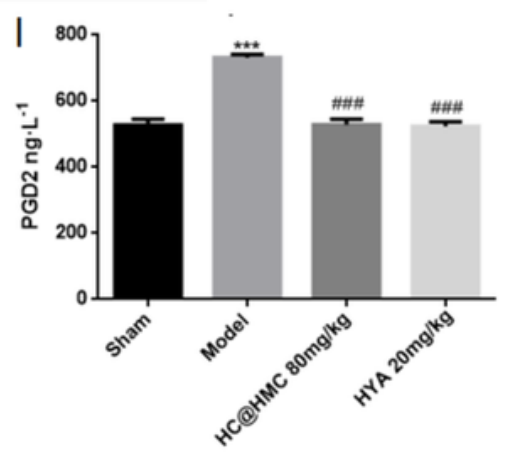

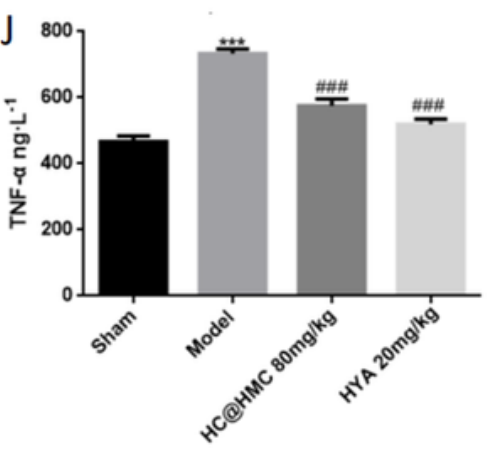

\section{Figure 5}

$\mathrm{HC} @ H M C$ attenuates inflammatory response in mice through COX-2/PGD2/DPs pathway. (A-C) Relative COX2 mRNA expressions and DP1 mRNA expressions and DP2 mRNA expressions in cortex after 24h of reperfusion ( $n=4) ;(D-F)$ Relative COX2 protein expressions and DP1 protein expressissons and DP2 protein expressions in cortex after $24 \mathrm{~h}$ of reperfusion $(n=4)$. (H-J) Changes of IL-1 $\beta \bigotimes P G D 2$, TNF-a content 
in cortex $(n=4)$. Data are presented as the mean $\pm S D$. ${ }^{*} P<0.05$, ${ }^{\star *} P<0.01$ and ${ }^{*} * *<<0.001$ compared with the sham group; $\# P<0.05$ and $\# \# P<0.01$ and \#\#\#P<0.001 compared with the model group.

\section{Supplementary Files}

This is a list of supplementary files associated with this preprint. Click to download.

- GraphicalAbstract.docx 